\title{
Cytogenetic testing in paediatrics: Some aspects of the Sri Lankan scenario
}

\author{
Vajira H W Dissanayake ${ }^{1}$, Rohan W Jayasekara ${ }^{2}$
}

Sri Lanka Journal of Paediatrics, 2008; 37: 38-41

(Key words: Cytogenetic testing, karyotyping, chromosome abnormalities)

Cytogenetic testing or chromosome culture and karyotyping was introduced to Sri Lanka in October 1983 with the establishment of the Human Genetics Unit of the Faculty of Medicine, University of Colombo. In 2006 a private sector laboratory too, entered the field. There has been an upward trend in the number of cytogenetic tests performed in Sri Lanka since 1983 (Figure 1). At the same time an unknown number of samples are sent abroad for testing.

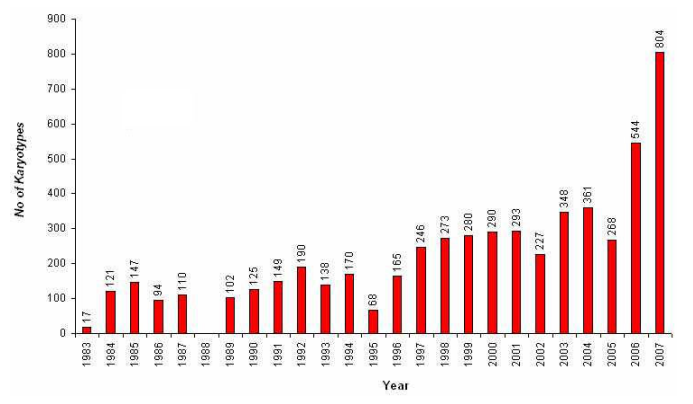

Figure 1 Cytogenetic Testing in Sri Lanka 1983 to 2007

1983 to 2005 - Data from the Human Genetics Unit, Faculty of Medicine, University of Colombo (HGU) 2006 to 2007 - Date from the HGU and the Genetic Laboratory, Asiri Surgical Hospital, Colombo Note: No tests were performed in 1988.

The first step in the process of helping these children and their families is establishing the correct genetic diagnosis of the affected child as illustrated in case 1 .

\section{Case 1}

In June 2007 the 40 year old wife of a 45 year old man delivered a baby with Down syndrome. They have two other normal children. The parents refused to accept that their baby had Down syndrome. The paediatrician ordered a karyotype on the affected baby. The karyotype was 46, XY, t(14;21) (figure 2).

The parents received genetic counselling. They accepted the diagnosis and understood what it meant for this baby and their other two children. They decided to undergo karyotyping. The wife's karyotype turned out to be 45, XX, t $(14 ; 21)$ (figure 3$)$.

${ }^{1}$ Senior Lecturer, Human Genetics Unit, ${ }^{2}$ Professor of Anatomy and Director Human Genetics Unit, Faculty of Medicine, University of Colombo.

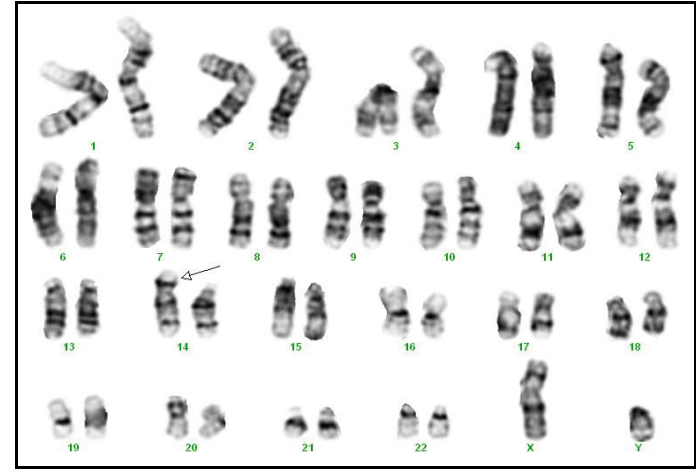

Figure 2 Karyogram of the baby at 450 band banding level showing an unbalanced translocation between chromosome no.14 and chromosome no.21. The karyotype is 46, XY, $t(14 ; 21)$.

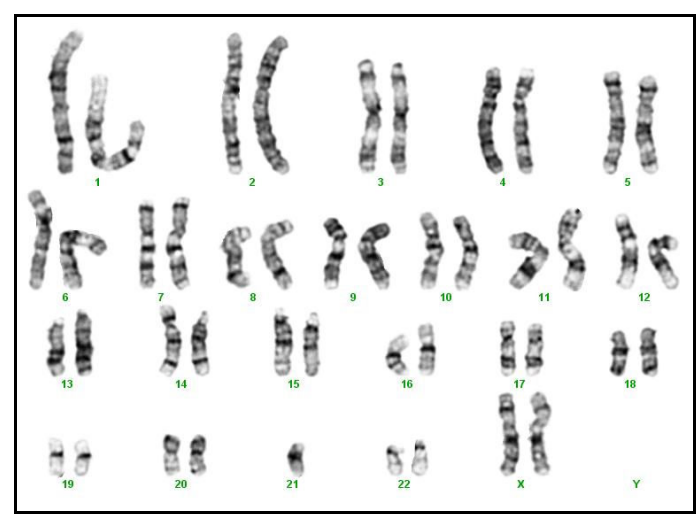

Figure 3 Karyogram of mother of the baby in figure 2 at 450 band banding level showing a balanced translocation between chromosome no.14 and chromosome no.21. Karyotype is 45, XX, t(14, 21).

She was a balanced translocation carrier. The couple had no plans to have more children. They decided to have a permanent method of contraception after discussing with an obstetrician/ gynaecologist. They also decided that they would tell their normal children about her carrier status when they become adults and advice them to find out whether they may be balanced translocation carriers. Furthermore they decided to convey their carrier status to the wife's younger sibling who was engaged to be married.

This family benefited because of a clinician who recognised the impact that a genetic diagnosis would have on the family and ordered the test. 
Unfortunately there are others who are under the misconception that a genetic diagnosis would not serve any purpose in situations like this because the parents are old, they anyway have two normal children, and therefore they are unlikely to want to have more children ${ }^{1}$.

Down syndrome (DS) is the commonest indication for karyotyping children in Sri Lanka. The causes of DS include: i) the presence of an extra chromosome no. 21 (trisomy 21); ii) presence of 2 cell lines - one has trisomy 21 and another has normal cells (mosaicism); and iii) the presence of a translocation of the long arm of chromosome no. 21 to usually chromosome nos. $13,14,15 ; 21$ or 22 (Table 1$)$

Table 1

The karyotype results of 615 consecutive babies with Down syndrome tested in Sri Lanka between 1 January 2002 and 31 December 2007

\begin{tabular}{|l|l|l|}
\hline \multicolumn{1}{|c|}{ Mechanism } & \multicolumn{1}{|c|}{ Karyotype } & No (\%) \\
\hline Non dysjunction & $\begin{array}{l}47, \mathrm{XX},+21 \text { or } \\
47, \mathrm{XY},+21\end{array}$ & $548(89.0)$ \\
\hline Mosaicism & $\begin{array}{l}47, \mathrm{XX},+21 / 46, \mathrm{XX} \text { or } \\
47, \mathrm{XY},+21 / 46, \mathrm{XY}\end{array}$ & $62(10.0)$ \\
\hline Translocation & $\begin{array}{l}46, \mathrm{XX}, \mathrm{t}(14 ; 21) \text { or } \\
46, \mathrm{XY}, \mathrm{t}(14 ; 21)\end{array}$ & $4(0.7)$ \\
& $46, \mathrm{XX}, \mathrm{t}(21 ; 21)$ & $1(0.0)$ \\
\hline
\end{tabular}

Translocations arise de novo during gametogenesis in normal parents or are constitutionally present in phenotypically normal balanced translocation carrier parents. The latter scenario increases the chance of recurrence of DS in the family; including in the cases of the $\mathrm{t}(21 ; 21)$ translocation, a recurrence risk of $100 \%{ }^{2}$. Among the families karyotyped because of DS in the index case or a family member in the past two years in our Unit, there were three families who had recurrent DS, i.e. more than one family member with DS. In all instances the tested person either had translocation DS or was a balanced translocation carrier. The extreme family concerns and social tensions that could be created by the recurrence of DS in a family due to the failure to offer genetic testing, counselling, and correct intervention at the appropriate time is illustrated in case 2 .

\section{Case 2}

In May 2007 a 28 year old woman was referred for genetic testing during her eighth pregnancy because she had given birth to several babies with DS. She had three pregnancies from her first marriage. The first pregnancy had resulted in a stillborn baby boy who had features of DS. The second pregnancy had resulted in the birth of a baby boy with DS who was 11 years old at the time of the referral. He had not been karyotyped. The third pregnancy had resulted in the birth of a baby girl who was reported to have died of complications of congenital heart disease at the age of 2 years and 6 months. She was not reported as having had DS. It was not possible to verify whether she in fact had DS because her medical records were not available as the woman had thrown them away in frustration. In her second marriage, the woman had three consecutive first trimester miscarriages. The obstetrician managing her eighth pregnancy, seeing her for the first time, had detected markers for DS on an ultrasound scan and referred her for karyotyping. She was karyotyped. Her karyotype was $45, X X, t(14 q ; 21 q) / 46 X X$. This indicated that she was mosaic for a cell line having a translocation involving chromosome nos. 14 and 21, and a normal cell line. She was counselled and referred back to the obstetrician.

A karyotype is useful to detect numerical and/or structural abnormalities in chromosomes. It can also detect mosaicism for such abnormalities. Karyotyping can be performed at different levels of banding resolution. Structural abnormalities not detected at the usual banding resolutions of 400 to 500 bands can be detected at higher banding resolutions of 600 or more (figure 4).

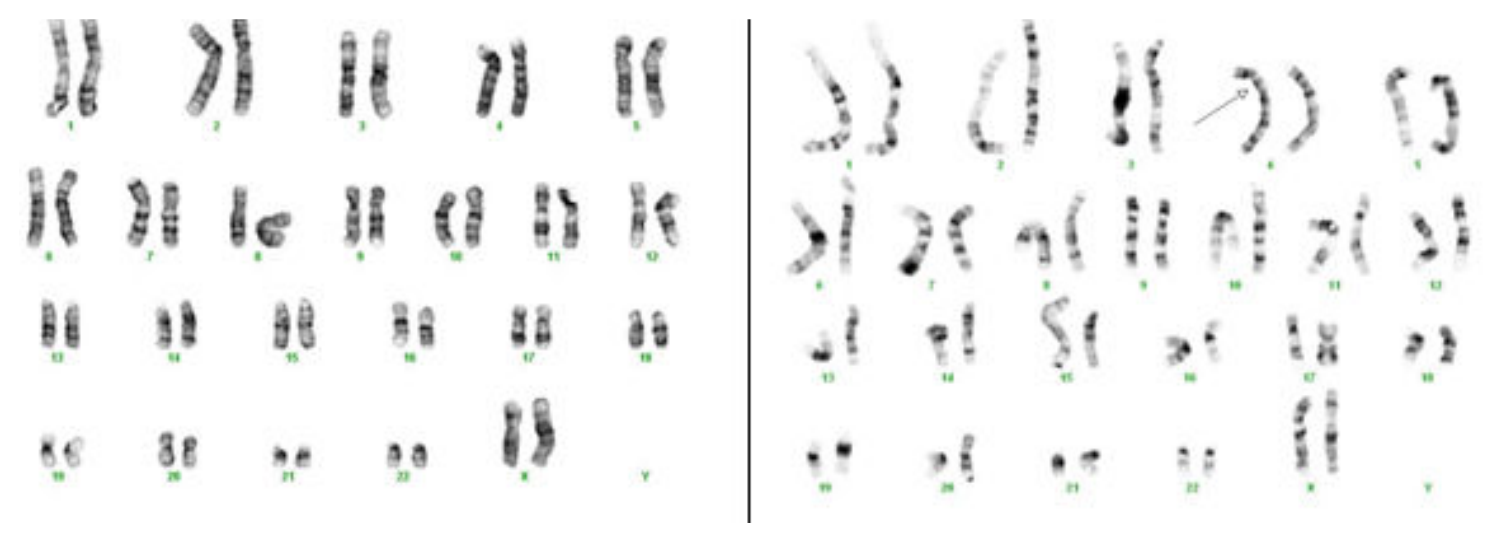

Figure 4 Two karyograms of the same patient. Karyogram on the left is at 450 band banding level. Karyogram on the right is at 550 band banding level. The duplication which is not clear on the karyogram on the left is clear on the karyogram on the right. The karyotype is 46, XX,dup(4) (q12-q21). 
This knowledge is especially important when one is dealing with subtle structural chromosome abnormalities. In any event chromosome culture and karyotyping, even when performed at standard banding resolutions, should be the starting point in the investigation of a suspected chromosome abnormality as illustrated in case 3 .

\section{Case 3}

In June 2007 a 2 years and 4 month old child with developmental delay, soft dysmorphic features and a distinctive "kitten-like" cry, the first child born to a young couple, was seen at a genetic consultation. In addition to that she had other features that were not part of the syndrome. Over the past two years various paediatricians attending on her had suspected cri-duchat (cat-cry) syndrome. They were advised by a specialist that it cannot be diagnosed in Sri Lanka. The parents however, wanted to get a second opinion on their own before they try to have another child and brought the baby for the consultation. The baby was karyotyped and was found to have a karyotype of 46,XX, der(5)add(8)(q13)del(5)(p13) (figure 5) indicating that she has features of cat-cry syndrome due to loss of the distal short arm of chromosome 5 .

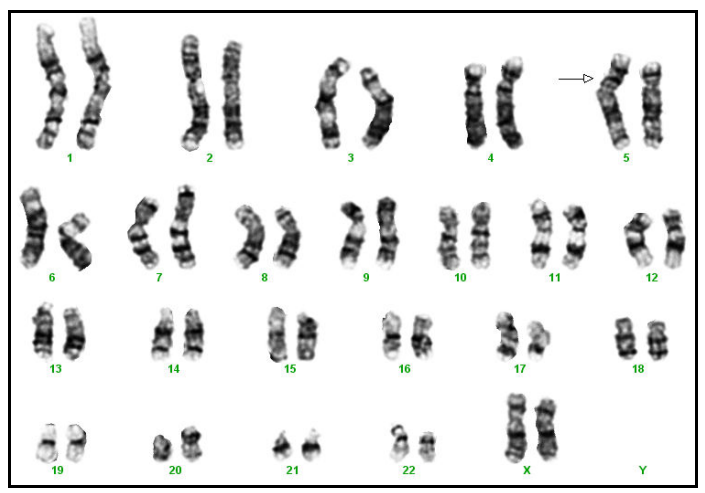

Figure 5 The karyogram of the baby at 450 band banding level showing a derivative chromosome no. 5 made up of parts of chromosome no. 5 and chromosome no. 8. The karyotype is 46, XX, der(5) add(8) (q13)del (5)(p13)

The additional features were possibly due to partial trisomy for distal long arm of chromosome 8. The parents were karyotyped. The father was found to be a balanced translocation carrier with the karyotype of $46, X Y, t(5 ; 8)(p 13 ; q 13)$ (figure 6).

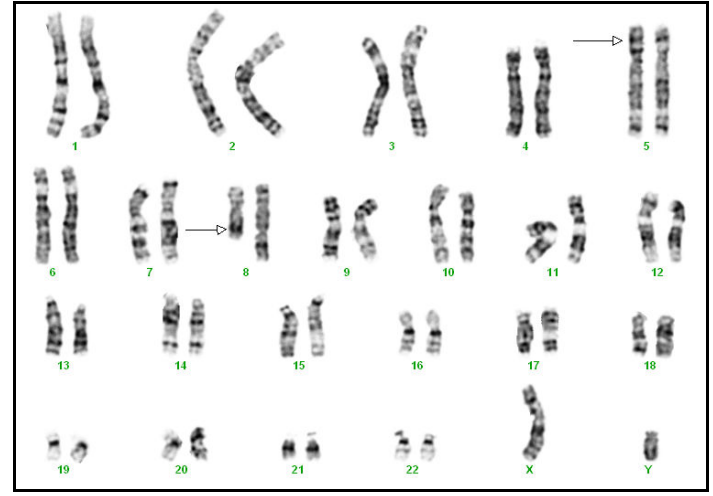

Figure 6 The karyogram of the phenotypically normal father of the baby with the chromosome abnormality shown in figure 5 at 550 band banding level showing a balanced translocation between chromosome no. 5 and chromosome no. 8. The karyotype is $46, X Y, t(5 ; 8)$ (p13;q13).

The family was counselled about the risk of recurrence of a chromosome abnormality in a future pregnancy and on the options they have to have a normal baby. They decided to try to have a normal baby by intrauterine insemination with donor sperms. They were referred to an obstetrician / gynaecologist.

At present there is no substitute for a karyotype to detect chromosome abnormalities in children. Usually a karyotype is prepared by analysing chromosomes found in chromosome spreads harvested from peripheral blood lymphocytes grown in culture for 6972 hours. Karyotyping is also performed on cells cultured from bone marrow, solid tissues, and amniotic fluid. Karyotyping amniotic fluid is time consuming as to do so amniocytes have to be grown in culture up to two weeks. To overcome this delay, several methods have been developed to screen amniotic fluid rapidly for the common numerical chromosome abnormalities found in antenatal amniotic fluid samples. Such tests screen for the presence of extra copies or loss of copies of chromosome no. 13, chromosome no. 18, chromosome no. 21, the $\mathrm{X}$ chromosome, and the $\mathrm{Y}$ chromosome. This is called aneuploidy screening. The methods used for aneuploidy screening include interphase fluorescence in situ hybridisation (FISH); short tandem repeat (STR) marker testing, and Multiple Ligation Probe Amplification (MLPA)3. It is not within the scope of this brief review to discuss the successful application of these techniques in antenatal screening. It would be sufficient however to state that such tests are not a substitute for karyotyping for genetic diagnosis in children as their diagnostic utility is limited. As illustrated below in case 4 and case 5 such tests can even give misleading or incorrect results. 


\section{Case 4}

In February 2005 a one month old child with ambiguous genitalia was referred for karyotyping by the paediatrician. While waiting for the karyotyping appointment, the parents inquired from another laboratory. The laboratory does not perform karyotyping but they perform a molecular genetic test for sex determination and a STR marker test for allele identification. Instead of informing the family that they do not perform karyotyping which was the genetic test indicated in this patient, they performed a combination of these tests on this child. Their report concluded as follows: "The presence of a 977 base pair DNA band indicates the presence of at least 1 copy of the X chromosome. The presence of a 788 base pair DNA band indicates the presence of the Y chromosome. This is a result expected from a male. Note: This test can not detect the presence of multiple copies of the X chromosome". The alert paediatrician immediately recognised that an improper test had been done and insisted on karyotyping. The child was karyotyped and found to have a normal male karyotype of 46, XY.

\section{Case 5}

In June 2006 an eight year old girl with short stature was referred for karyotyping because the paediatrician suspected Turner Syndrome. Since the appointment for karyotyping was in six weeks time, the parents inquired from the same laboratory as in case 4 . This contact resulted in another inappropriate test which concluded as follows: "The above tests indicate that the tested individual does not have a $\mathrm{Y}$ chromosome and has only one copy of the $\mathrm{X}$ chromosome". The alert paediatrician immediately recognised that an improper test had been done and insisted on karyotyping. She was karyotyped and found to have a normal female chromosome complement of $46, \mathrm{XX}$.

Chromosome culture and karyotyping is not a luxury anymore. It is an essential investigation in the armoury of diagnostic tools available to the paediatrician. Paediatricians should be aware of the diagnostic use of chromosome culture and karyotyping. They should also be aware that rapid aneuploidy screening methods should not be used in routine cytogenetic diagnosis in children. This knowledge would help them to direct their patients to the correct laboratory for the indicated genetic test. By doing so, in addition to avoiding unnecessary tests, they can help save money for the parents/guardians as genetic testing is not free in Sri Lanka.

This article was confined to examples on the use of karyotyping to diagnose dysmorphic conditions. Cytogenetics however is also becoming indispensable in the management of haematological malignancies in childhood4. Karyotyping as well as chromosome breakage studies are important in the diagnosis of haematological malignancies. All these tests are now available locally.
The above cases also highlight the complicated ethical problems that genetic testing is associated with because of the special nature of genetic information, i.e. that the test information derived from one individual may have implications for others in the family. It is important therefore, that pre- and post-test counselling is offered to families undergoing genetic testing, and that genetic testing be carried out only after obtaining written informed consent. These should be a part of the services offered by any genetic laboratory, and clinicians should not hesitate to make use of such services.

Case 5 also highlights the potential legal ramifications of the use of an inappropriate test. The social stigma attached to a genetic diagnosis is immense. As such no one should be wrongly labelled with a genetic diagnosis. Both laboratories conducting genetic testing as a clinical genetic diagnostic service and clinicians ordering genetic tests should be aware that there is no substitute for chromosome culture and karyotyping to diagnose chromosome abnormalities. Their responsibilities are not mutually exclusive. We would like to recommend that, to avoid such pitfalls, professional medical associations such as the Sri Lanka College of Paediatricians draw up guidelines on appropriate use of genetic testing for their members. In the long term however, regulatory mechanisms, such as those proposed in the New Genetics and Assisted Reproductive Technologies in Sri Lanka, A Draft National Policy on Bioethics written by a committee set up by the National Science and Technology Commission of Sri Lanka in 2003 comprising of paediatricians, obstetrician gynaecologists, geneticists and lawyers, would have to be implemented5. Such action is currently under consideration by the National Bioethics Commission of the National Science Foundation, Sri Lanka.

\section{References}

1. de Silva D. Emerging issues in genetic testing in Sri Lanka. Indian J Med Ethics 2007; 15(3):133.

2. Gardner R J M, Sutherland G R. Down Syndrome, other full aneuploidies, and polyploidy. In Chromosome abnormalities and genetic counselling. New York: Oxford University Press, 1996: 243-58.

3. Shaffer L G, Bui T H, Molecular cytogenetic and rapid aneuploidy detection methods in prenatal diagnosis. Am J Med Genet C Semin Med Genet, 2007; 145(1): 87-98.

4. Johansson B, Mertens F, Mitelman F. Clinical and biological importance of cytogenetic abnormalities in childhood and adult acute lymphoblastic leukaemia. Ann Med 2004; 36(7):492-503.

5. National Science and Technology Commission, Sri Lanka. New Genetics and Assisted Reproductive Technologies in Sri Lanka, A Draft National Policy on Bioethics (NTSC-SG-09/03/01). 2003. NASTEC, Colombo. 